\title{
On marginally resolved objects in optical interferometry
}

\author{
R. Lachaume ${ }^{\star}$ \\ Observatoire de Grenoble, Laboratoire d'Astrophysique de Grenoble, Université Joseph Fourier, BP 53, 38041 Grenoble Cedex, \\ France \\ Received 13 November 2002 / Accepted 19 December 2002

\begin{abstract}
With the present and soon-to-be breakthrough of optical interferometry, countless objects shall be within reach of interferometers; yet, most of them are expected to remain only marginally resolved with hectometric baselines.

In this paper, we tackle the problem of deriving the properties of a marginally resolved object from its optical visibilities. We show that they depend on the moments of flux distribution of the object: centre, mean angular size, asymmetry, and curtosis. We also point out that the visibility amplitude is a second-order phenomenon, whereas the phase is a combination of a first-order term, giving the location of the photocentre, and a third-order term, more difficult to detect than the visibility amplitude, giving an asymmetry coefficient of the object. We then demonstrate that optical visibilities are not a good model constraint while the object stays marginally resolved, unless observations are carried out at different wavelengths. Finally, we show an application of this formalism to circumstellar discs.
\end{abstract}

Key words. methods: data analysis - techniques: interferometric

\section{Introduction}

Optical systems are fundamentally limited in angular resolution by their spatial extent because of diffraction. Very soon, the idea of combining light coming from two "distant" telescopes was investigated in order to overcome the limitation in size of single pupils. Michelson (1891, 1920), Michelson $\&$ Pease (1921) derived the angular diameters of some solarsystem bodies and stars by measuring the contrast of the fringes (called visibility amplitude) obtained when interfering light comes from two apertures: this contrast is maximum when these apertures are closest and decreases with the distance between telescopes (called the baseline). The baseline at which the fringes disappear holds information on the angular extent of the source. After some time, the mid-seventies saw the come-back of optical interferometry with Hanbury Brown et al. (1974), Labeyrie (1975), but it long stayed confined to bright and simple objects, mostly stellar diameters and multiple systems. It is all the more frustrating as the theory of interferometry allows image reconstruction and as radio arrays achieved this goal within a few decades of existence: the atmospheric turbulence and the nature of light both lead to complex optical designs and have slowed the development of optical interferometry. Moreover, the shift of the fringes (called phase) is completely blurred by the atmosphere, so techniques to retrieve phase information - necessary for imaging capacities - needed additional investigation.

\footnotetext{
* e-mail: Regis.Lachaume@obs.ujf-grenoble.fr
}

Recently, the Palomar Testbed Interferometer (PTI, Colavita et al. 1999) and the Infrared Optical Telescope Array (IOTA, Carleton et al. 1994) allowed us to probe circumstellar matter in star-forming regions (Malbet et al. 1998; Akeson et al. 2000; Malbet \& Berger 2002b; Akeson et al. 2002), giving some constraints on the geometry of these objects. With the advent of the Very Large Telescope Interferometre (VLTI, Glindemann et al. 2000) and the (KI, Colavita 2001) we are expecting a much higher accuracy with their large pupils (8$10 \mathrm{~m}$ ), and good constraints on objects thanks to the number of baselines available and partial phase information; yet they will not allow direct image reconstruction very soon because recombination will be first performed with two or three telescopes. The Navy Prototype Optical Interferometre (NPOI, Armstrong et al. 1998), the CHARA array (ten Brummelaar et al. 2000), and the Cambridge Optical Aperture Synthesis Interferometer (COAST, Haniff et al. 2000) provide imaging capacities with a multi-telescope recombination, but with a lower sensitivity that renders faint object science difficult.

We are clearly entering a phase in which more than an apparent diameter is measured but no imaging is performed; in this context, observers and modellers use interferometric observations as a constraint on models (e.g. Malbet \& Berger 2002b; Akeson et al. 2002; Lachaume et al. 2003), but their link with the geometry of the object remains unclear; it is still quite common to think in terms of diameter. For instance, Monnier \& Millan-Gabet (2002) link the uniform disc equivalent diameter derived from the IR interferometric observations of young stellar objects with the physical radius of their supposed inner hole. The phase also raises problems of geometrical 
interpretation. Since it is blurred by the atmosphere, one uses the closure phase to retrieve partial information on it $^{1}$ : the principle is to add the phases over a triplet of baselines provided by three telescopes, which allows one to cancel atmospheric terms. It is generally used either in image reconstruction, at NPOI for instance, or as a model constraint. Geometrically speaking, it is a diagnosis of asymmetry, but its accurate meaning is seldom made clear enough.

In this paper, we connect the visibility amplitude and phase with the geometry of the object, which allows us to retrieve information in a model-independent fashion. In Sect. 2, we establish a series development of these quantities involving the moments of the flux distribution, the first ones being the location of the photocentre, the spatial extent (diameter), and the asymmetry coefficient (skewness). It appears as a generalisation of the widespread diameter measurement. We then draw the consequences of the formalism in terms of observation and modelling. In Sect. 3 we apply this development to circumstellar discs with two examples: the case of an object characterised by more than one diameter (star, thermal flux and scattered light) and the measurement of the radial temperature law in these discs.

\section{Visibility and object geometry}

The Zernicke-van Cittert theorem links the complex visibility $V$ to the normalised flux distribution $I$ of the object:

$V(\boldsymbol{u})=\iint I(\alpha) \exp (-2 \pi i \boldsymbol{u} \cdot \boldsymbol{\alpha}) \mathrm{d}^{2} \boldsymbol{\alpha}$,

where $\boldsymbol{u}$ is linked to the projected baseline $\boldsymbol{B}$ and the wavelength $\lambda$ by $\boldsymbol{u}=\lambda^{-1} \boldsymbol{B}$, and $\boldsymbol{\alpha}$ the angular location on the sky. Optical interferometry usually deals with the square amplitude $|V|^{2}$ and the phase $\phi$, given by

$$
\begin{aligned}
|V|^{2} & =(\operatorname{Re} V)^{2}+(\operatorname{Im} V)^{2}, \\
\tan \phi & =(\operatorname{Im} V) /(\operatorname{Re} V) .
\end{aligned}
$$

In interferometry two extreme cases are usually dealt with: on the one hand, an object is fully resolved if its angular size is of the order of $B / \lambda$ as it would be with a single dish telescope of size $B$. In that case, the visibility is arbitrary and highly depends on the shape of the target. On the other hand, a point-like source is not resolved and its visibility is $V=1$. Between these two cases, the object is said to be marginally resolved and $V$ is close to unity; its size is a fraction of $B / \lambda$. In such a case, most of the flux is located in a zone where $|\boldsymbol{u} \cdot \boldsymbol{\alpha}| \ll 1$, so we carry out a series development to derive the real and imaginary parts of $V$ :

$$
\begin{aligned}
& \operatorname{Re} V(u)=\iint I(\boldsymbol{\alpha})\left(1-2 \pi^{2}(\boldsymbol{u} \cdot \boldsymbol{\alpha})^{2}\right) \mathrm{d}^{2} \boldsymbol{\alpha}, \\
& \operatorname{Im} V(u)=\iint I(\boldsymbol{\alpha})\left(2 \pi(\boldsymbol{u} \cdot \boldsymbol{\alpha})-\frac{4}{3} \pi^{3}(\boldsymbol{u} \cdot \boldsymbol{\alpha})^{3}\right) \mathrm{d}^{2} \boldsymbol{\alpha} .
\end{aligned}
$$

\footnotetext{
${ }^{1}$ Other techniques are also the use of a close object as a phase reference or the differential phase with spectroscopy, but we shall not discuss them in this paper.
}

These expressions drive us to define the $n$th moment of the flux distribution as a symmetric tensor unambiguously defined by

$$
M_{n} \cdot \boldsymbol{u}_{1} \cdots \boldsymbol{u}_{n}=\iint I(\alpha)\left(\alpha \cdot \boldsymbol{u}_{1}\right) \cdots\left(\alpha \cdot \boldsymbol{u}_{n}\right) \mathrm{d}^{2} \boldsymbol{\alpha}
$$

for any set of vectors $\boldsymbol{u}_{1}, \cdots, \boldsymbol{u}_{n}$. (The expressions for the first two moments are given in a Cartesian frame in Appendix A.) With such a definition, the real and imaginary parts of $V$ become

$$
\begin{aligned}
& \operatorname{Re} V(u)=1-2 \pi^{2} \boldsymbol{M}_{2} \cdot \boldsymbol{u} \cdot \boldsymbol{u} \\
& \operatorname{Im} V(u)=2 \pi \boldsymbol{M}_{1} \cdot \boldsymbol{u}-\frac{4}{3} \pi^{3} \boldsymbol{M}_{3} \cdot \boldsymbol{u} \cdot \boldsymbol{u} \cdot \boldsymbol{u}
\end{aligned}
$$

which, after a few calculations, give

$$
\begin{aligned}
|V|^{2}= & 1-4 \pi^{2}\left[\boldsymbol{M}_{2} \cdot \boldsymbol{u} \cdot \boldsymbol{u}-\left(\boldsymbol{M}_{1} \cdot \boldsymbol{u}\right)^{2}\right] \\
\phi= & -2 \pi\left(\boldsymbol{M}_{1} \cdot \boldsymbol{u}\right)+\frac{4}{3} \pi^{3}\left[\boldsymbol{M}_{3} \cdot \boldsymbol{u} \cdot \boldsymbol{u} \cdot \boldsymbol{u}\right. \\
& \left.-3\left(\boldsymbol{M}_{1} \cdot \boldsymbol{u}\right)\left(\boldsymbol{M}_{2} \cdot \boldsymbol{u} \cdot \boldsymbol{u}\right)+2\left(\boldsymbol{M}_{1} \cdot \boldsymbol{u}\right)^{3}\right]
\end{aligned}
$$

These formulae are quite annoying because the visibility amplitude apparently depends on the location of the photocentre of the object, given by the first moment $\boldsymbol{M}_{1}$ (see Appendix A), and therefore on the pointing accuracy of the instrument. In order to cancel this apparent dependence, we define the moments in respect to the photocentre of angular location $\alpha_{0}$ by

$\boldsymbol{M}_{n}^{\prime} \cdot \boldsymbol{u}_{1} \cdots \boldsymbol{u}_{n}=\iint I(\alpha)\left(\alpha-\alpha_{0}\right) \cdot \boldsymbol{u}_{1} \cdots\left(\alpha-\alpha_{0}\right) \cdot \boldsymbol{u}_{n} \mathrm{~d}^{2} \boldsymbol{\alpha}$

and perform a frame change on Eqs. $(6 a, 6 b)$ to obtain

$$
\begin{aligned}
|V|^{2} & =1-4 \pi^{2}\left(\boldsymbol{M}_{2}^{\prime} \cdot \boldsymbol{u} \cdot \boldsymbol{u}\right)+O\left(u^{4}\right) \\
\phi & =-2 \pi\left(\boldsymbol{M}_{1} \cdot \boldsymbol{u}\right)+\frac{4}{3} \pi^{3}\left(\boldsymbol{M}_{3}^{\prime} \cdot \boldsymbol{u} \cdot \boldsymbol{u} \cdot \boldsymbol{u}\right)+O\left(u^{5}\right) .
\end{aligned}
$$

In some applications, a higher-order development is needed; it is given in Appendix B.

Since the phase is not directly measured because of atmospheric turbulence, the closure phase is used instead. With three telescopes labelled 1,2 , and 3 simultaneously providing the baselines $\boldsymbol{u}_{12}, \boldsymbol{u}_{23}$, and $\boldsymbol{u}_{31}$, the closure phase reads

$\bar{\phi}=\phi\left(\boldsymbol{u}_{12}\right)+\phi\left(\boldsymbol{u}_{23}\right)+\phi\left(\boldsymbol{u}_{31}\right)$.

Applying Eq. (8b), we derive a concise expression:

$\bar{\phi}=\frac{4}{3} \pi^{3}\left(\boldsymbol{M}_{3}^{\prime} \cdot \boldsymbol{u}_{12} \cdot \boldsymbol{u}_{23} \cdot \boldsymbol{u}_{31}\right)+O\left(u^{5}\right)$

\subsection{Resolving an object: Size, asymmetry and curtosis}

We consider three aligned telescopes labelled from 1 to 3 , providing the baselines $\boldsymbol{u}_{12}, \boldsymbol{u}_{23}$, and $\boldsymbol{u}_{31}$ in a direction given by a normal vector $\boldsymbol{i}$, as represented in Fig. 2. The main characteristics of an object we shall consider are its mean diameter, its 

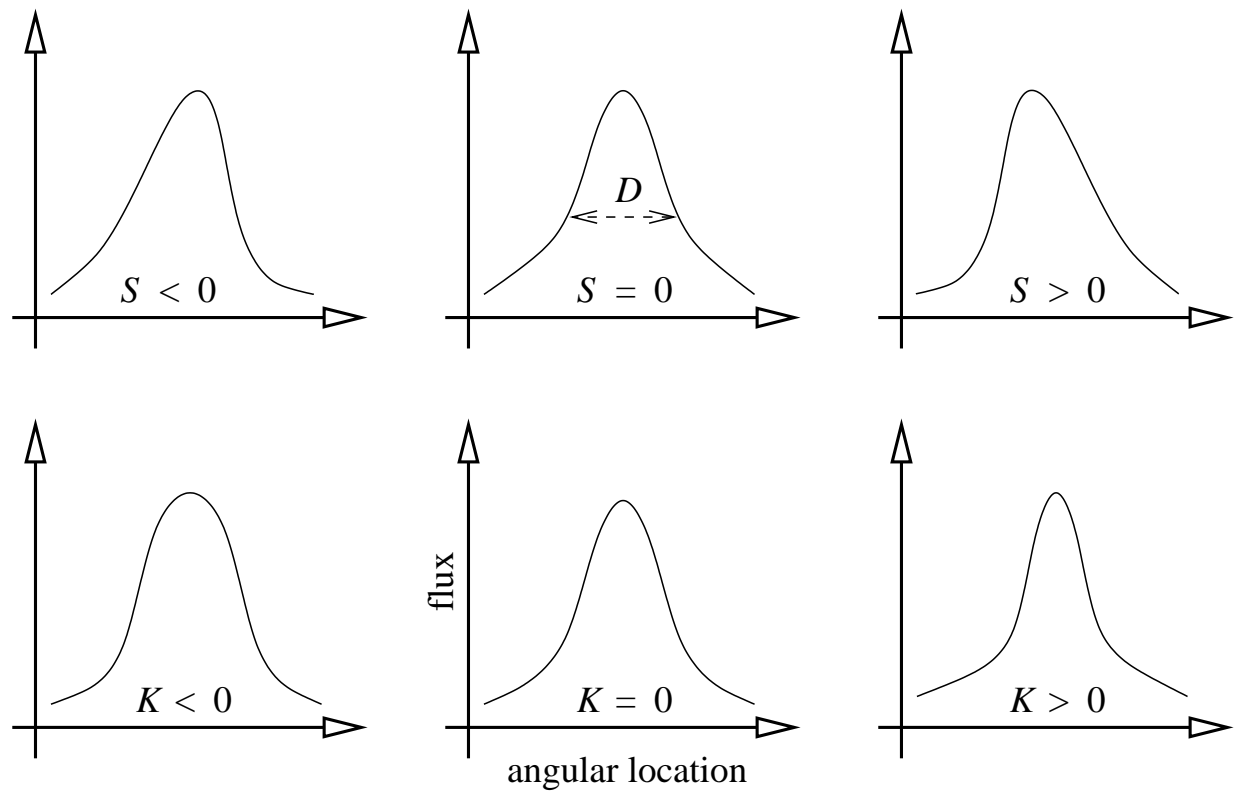

Fig. 1. Link between the shape of a flux distribution and its first moments: mean diameter $D$, asymmetry coefficient $S$ (skewness), and curtosis $K$.

asymmetry and its curtosis defined along $i$; they respectively are

$$
\begin{aligned}
D & =\sqrt{M_{2}^{\prime} \cdot \boldsymbol{i} \cdot \boldsymbol{i}}, \\
S & =\frac{M_{3}^{\prime} \cdot \boldsymbol{i} \cdot \boldsymbol{i} \cdot \boldsymbol{i}}{\left(M_{2}^{\prime} \cdot \boldsymbol{i} \cdot \boldsymbol{i}\right)^{3 / 2}}, \\
K & =\frac{M_{4}^{\prime} \cdot \boldsymbol{i} \cdot \boldsymbol{i} \cdot \boldsymbol{i} \cdot \boldsymbol{i}}{\left(M_{2}^{\prime} \cdot \boldsymbol{i} \cdot \boldsymbol{i}\right)^{2}}-3 .
\end{aligned}
$$

They are of common use in statistics. Figure 1 displays their link with the shape of a one dimensional distribution: the diameter $D$ is the square root of the variance, giving the mean spatial extent; the skewness $S$ increases with the asymmetry of the distribution and is zero for a symmetrical one; $K$ indicates whether the flux is concentrated in the peak of the distribution or in its wings and is zero for a normal distribution. With these notations,

$$
\begin{aligned}
|V(u)|^{2} & =1-4 \pi^{2}(D u)^{2}+\frac{4}{3} \pi^{4}\left[(K+6)(D u)^{4}\right], \\
\bar{\phi} & =\frac{4}{3} \pi^{3} S(D \bar{u})^{3},
\end{aligned}
$$

where the mean baseline $\bar{u}$ is given by the geometrical mean of the three baselines:

$\bar{u}=\sqrt[3]{\left(\boldsymbol{u}_{12} \cdot \boldsymbol{i}\right)\left(\boldsymbol{u}_{23} \cdot \boldsymbol{i}\right)\left(\boldsymbol{u}_{31} \cdot \boldsymbol{i}\right)}$.

A summary of these results, in a less formal way, is given in Fig. 2.

The main implication of these results is that the visibility amplitude drop is a second-order phenomenon $\left(D^{2} u^{2}\right)$ while the closure phase is a third-order one $\left(S D^{3} u^{3}\right)$. As a consequence, closure phase is much harder to detect than the visibility amplitude in a marginally resolved object. Figure 3 displays the profile of the visibility amplitude and closure phase as a function of the baseline for a marginally resolved object with

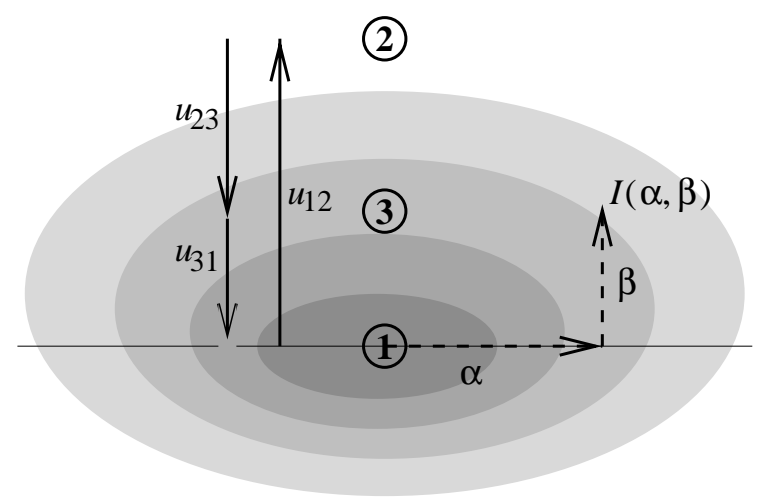

$$
\begin{aligned}
\left|V_{i j}\right|^{2} & =1-4 \pi^{2} u_{i j}^{2} \underbrace{\iint I(\alpha, \beta) \beta^{2} \mathrm{~d} \alpha \mathrm{d} \beta}_{D^{2}} \\
\bar{\phi} & =\frac{4}{3} \pi^{3} \underbrace{u_{12} u_{21} u_{31}}_{\bar{u}^{3}} \underbrace{\iint I(\alpha, \beta) \beta^{3} \mathrm{~d} \alpha \mathrm{d} \beta}_{S D^{3}}
\end{aligned}
$$

Fig. 2. Link between the flux distribution $I(\alpha, \beta)$, the visibility amplitude $|V|$, and the closure phase $\bar{\phi}$ for a marginally resolved object. The three aligned telescopes, numbered 1 to 3 , and the baselines are projected onto the sky. Third-order terms and lower have been kept.

a high asymmetry $S=0.5$, as well as the minima of detection for these quantities as a function of the measurement accuracy. It appears that the asymmetry is detected for angular sizes 3 to 6 times larger than the spatial extent or - which is equivalent for 3 to 6 times larger baselines.

\subsection{Validity of the approximation}

The above development presents two limitations: on the one hand, it assumes that all moments are defined and, on the other 

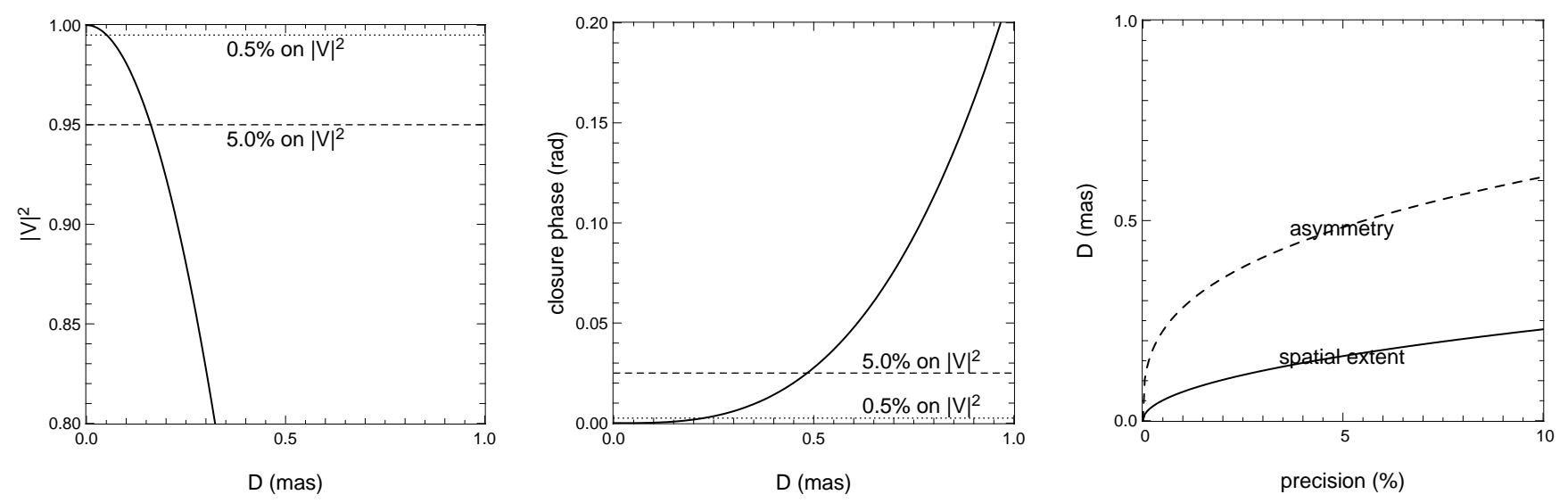

Fig. 3. Variation of the square visibility amplitude and closure phase with the angular size of a marginally resolved object for a $100 \mathrm{~m}$ baseline in $\mathrm{K}$, and the detectability of these quantities. Left panel: square visibility amplitude vs. angular size; middle panel: closure phase vs. angular size; right panel: minimum object size needed to detect either the spatial extent or the asymmetry vs. the instrumental precision on $|V|^{2}$. On the first left two view graphs the detection levels for $0.5 \%$ and $5 \%$ accuracy on $|V|^{2}$ measurements are displayed. We assumed a fairly asymmetric object with $S=0.5$, as well as $\Delta \bar{\phi}=\Delta V$.
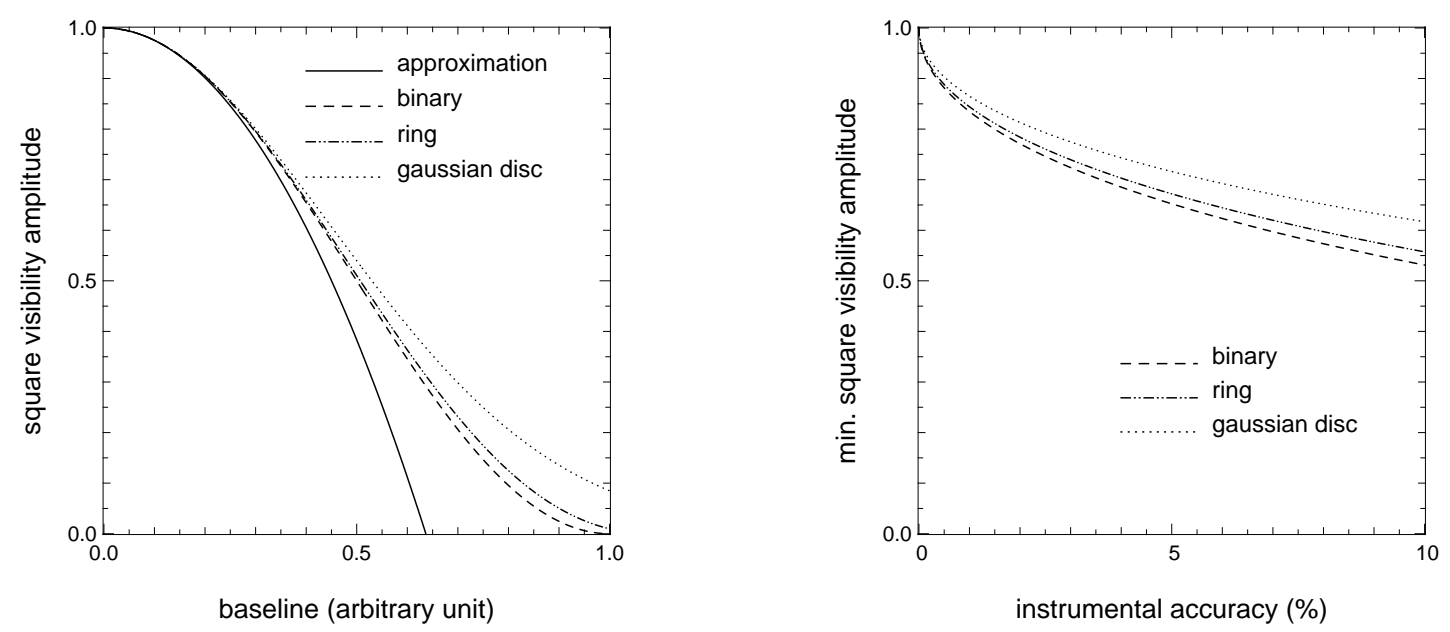

Fig. 4. Comparison between the exact visibility amplitude of an object and its second-order estimate. Different geometries have been assumed to show that little dependence is found: a symmetrical binary (dashes), a ring (dash-dot-dot), and a Gaussian disc (dots). The left panel displays the second-order estimate (solid line) and the exact visibilities of the different objects vs. the baseline. The right panel displays the visibility amplitude for which an observational difference can be made between the estimate and the exact value vs. the instrumental precision; it is the limit of validity for this estimate.

hand, the first few terms of the series are no longer a good approximation when the object is resolved enough.

In the case of a power law distribution $I(\alpha) \propto \alpha^{-q}$, often encountered when scattered light dominates, the high-order moments are not defined. Therefore the above development is no longer valid. For instance, Lachaume et al. (2003) have shown that a disc with scattering presents a quick drop of the visibility amplitude near the origin $u=0$, that definitely does not present the smooth profile $|V|^{2}=1-4(\pi D u)^{2}$. In Sect. 3.1, we shall see how to treat scattering at a large scale, while using the above formalism for other flux contributions.

Another important point is the range of validity. The left panel of Fig. 4 compares the exact visibility of different types of objects with the second-order estimate (first terms of Eq. (14)) as a function of the baseline: as expected, the approximation is correct for under resolved objects with $V \approx 1$ but gets poorer with larger baselines. The validity of the approximation depends on whether a difference can be made between the estimate and the exact value, in other terms whether the accuracy of the measurement is better than the precision of the estimate. The right panel of the same figure displays the visibility at which the instrument accuracy allows us to measure the deviation from the estimate; it appears not to be much dependent on the geometry of the object. With a typical $2 \%$ accuracy on $|V|^{2}$, the estimate is valid for $|V|^{2} \gtrsim 0.8$.

Figure 5 is similar to Fig. 4 but for the closure phase. For a typical binary with $\Delta .2$, it displays the exact closure phase and the third order estimate given in Eq. (15) as a function of the baseline. The right panel indicates the visibility at which a differentiation between them can be made as a function of the instrumental accuracy. With the typical $2 \%$ precision on $|V|^{2}$, the estimate of the phase is valid for $|V|^{2} \gtrsim 0.45$.

When the accuracy allows us to see the deviation from the third-order estimates, some following orders can be 

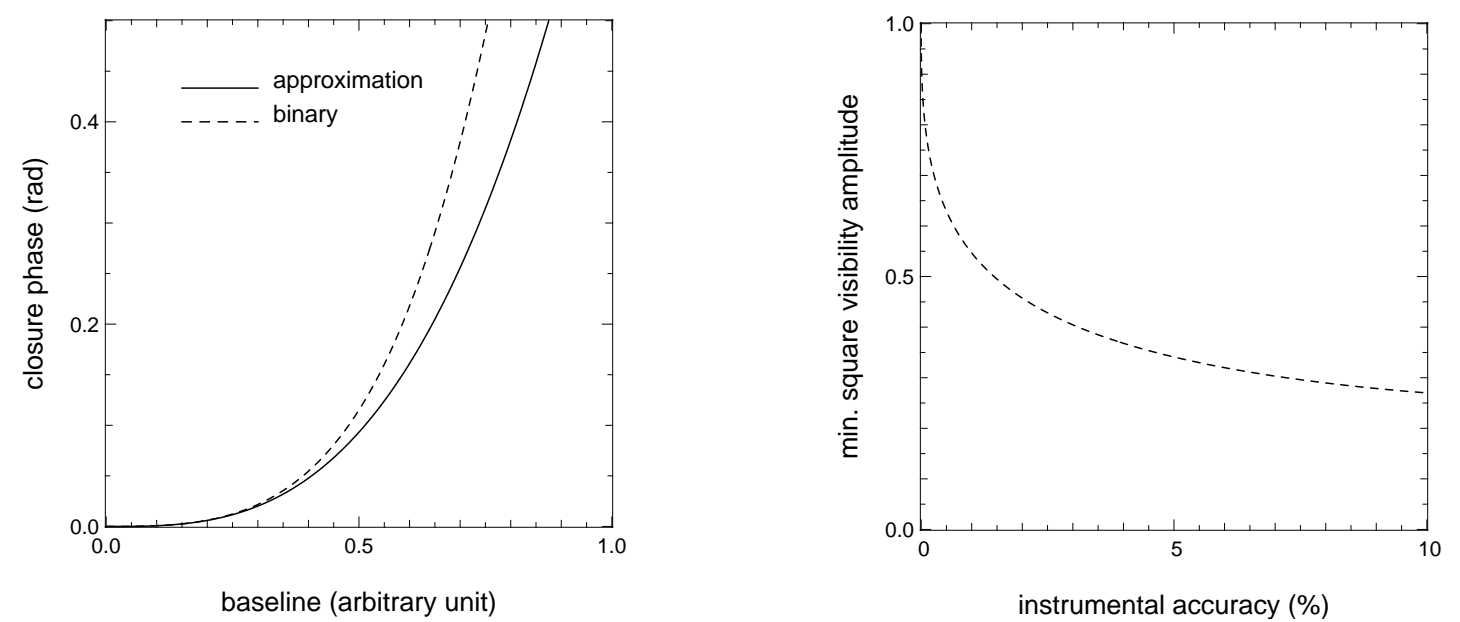

Fig. 5. Comparison between the exact closure phase of an asymmetrical binary $(\Delta \operatorname{mag}=1.2)$ and its third-order estimate. Left panel: closure phase vs. mean baseline. Right panel: minimum visibility for which no observational difference can be made between the estimate and the exact phase vs. instrument accuracy.

measured and the subsequent moments of the distribution can be accessed. This can be a means to retrieve model-independent spatial information.

\subsection{Model-fitting of observations}

Theoretically speaking, the knowledge of visibility and closure phase at all baselines smaller than $B$ would allow image reconstruction with an infinite resolution (by using the analycity of the visibility); so, marginal resolution should not be a problem. This point presents a small but irretrievable flaw: it assumes that there is no noise in the data. When the finite precision of measurements is taken into account, the accuracy on the reconstructed image or model is impaired and also, the number of parameters constrained in a marginally resolved object. If the visibility amplitude alone is available, only the quadratic form $V^{2}=1-4 \pi^{2} \boldsymbol{M}_{2}^{\prime} \cdot \boldsymbol{u} \cdot \boldsymbol{u}$ can be accessed, because the deviation from this law cannot be measured, as shown in Sect. 2.2.

We first consider an observation carried out at a single wavelength and use the coordinates $(u, v)$ of $\boldsymbol{u}$ in a Cartesian frame. The visibility amplitude and phase then are

$V=1-4 \pi^{2}\left(M_{2}^{\prime}{ }_{2}^{2}+2 M_{2}^{\prime}{ }_{2}^{1} u v+M_{2}^{\prime 2} v^{2}\right)$,

$\phi=1-\frac{4}{3} \pi^{3}\left(M^{\prime 0}{ }_{3} u^{3}+3 M^{\prime 1}{ }_{3}^{2} v+3 M^{\prime 2}{ }_{3} u v^{2}+M^{\prime}{ }_{3}^{3} v^{3}\right)$,

where $M_{n}^{\prime k}$ is a component of the $n$th moment $\boldsymbol{M}_{n}^{\prime}$. Its expression in the Cartesian frame can be found in Appendix A. If visibility amplitude alone is considered, the system can be described with three parameters (the $M_{2}^{\prime k}$ ); a model able to fit any second-order moment will fit any data. As a consequence, a marginally resolved object observed in visibility amplitude, whatever the baseline coverage is, can be modelled by

- a ternary system of unresolved stars;

- a Gaussian elliptic disc;

- a uniform stellar photosphere and an unresolved star.

If closure phase information is available, then four additional parameters describe the object (the $M_{3}^{\prime k}$ ). A marginally resolved object observed in both visibility and closure phase can be modelled by a system able to reproduce the second- and third-order moments, that is

- a system of four unresolved stars;

- a Gaussian elliptic disc and an unresolved star;

- a uniform stellar photosphere and two unresolved stars.

It appears that interferometry does not allow us to disentangle quite different scenarios when the object is marginally resolved. This fact is well-known by observers: under resolved observations cannot distinguish between a uniform and a limbdarkened stellar disc; neither could the first observations of FU Ori by Malbet et al. (1998) exclude either the disc or the binary scenario. As a remedy, one can use the technique in combination with other types of observations, as another model constraint.

Nevertheless, a multi-wavelength interferometric approach can bring more constraints. We consider a $N_{\mathrm{p}}$ point-like source model to be fitted to visibility amplitudes at $N_{\lambda}$ wavelengths. The fluxes and locations of the $N_{\mathrm{p}}-1$ first sources constrain the last one, because the flux distribution is normalised and centered; therefore there are $\left(N_{\mathrm{p}}-1\right)$ locations and $N_{\lambda}\left(N_{\mathrm{p}}-1\right)$ fluxes, that is $\left(N_{\lambda}+2\right)\left(N_{\mathrm{p}}-1\right)$ free parameters. Observations provide $3 N_{\lambda}$ moments of the flux distribution. The characteristic number of point-like sources constrained by the measurements is given by the equality between the number of free parameters and that of moments, so that

$N_{\mathrm{p}}=\left\lceil\frac{3 N_{\lambda}}{N_{\lambda}+2}+1\right\rceil$

The left panel of Fig. 6 displays the number of sources constrained as a function of the number of wavelengths accessed, either observing visibility amplitudes only or both amplitudes and closure phases. It appears that amplitudes and phases can constrain up to 6 point-like sources with $N_{\lambda} \gtrsim 10$. If we now consider that the sources emit a black-body spectrum - or whatever spectrum determined by a temperature and 

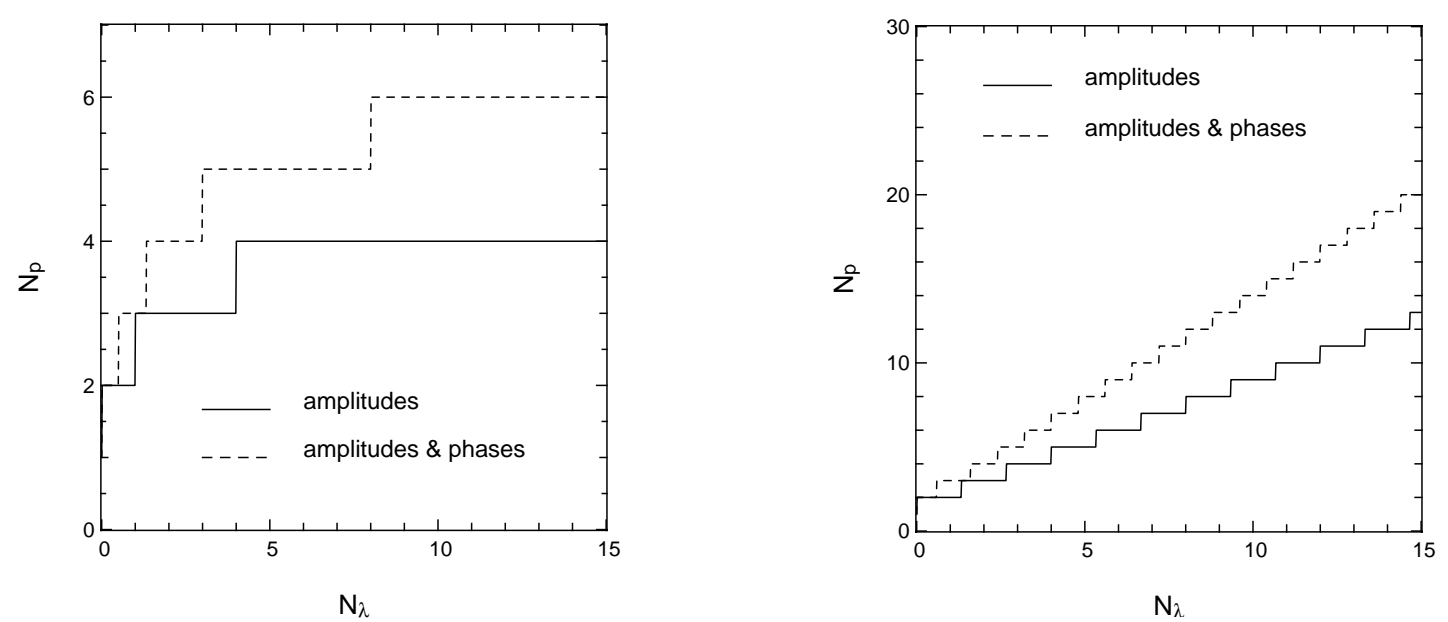

Fig. 6. Point-like source model fitting: number of sources constrained by interferometric observations of a marginally resolved object vs. number of wavelengths used. Left panel: the fluxes at different wavelengths are not correlated. Right panel: the sources are assumed to emit a black-body spectrum.

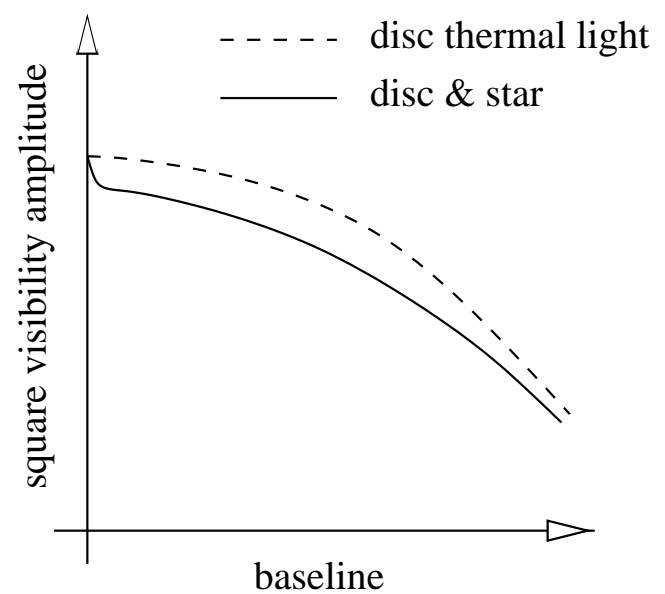

Fig. 7. Visibility curve of an accretion disc. Solid line: all contributions; dashed line: thermal emission of the disc only.

a bolometric flux - there are only $2\left(N_{\mathrm{p}}-1\right)$ fluxes. The number of point-like sources constrained then becomes

$N_{\mathrm{p}}=\left\lceil\frac{3 N_{\lambda}}{4}+1\right\rceil$.

The right panel of Fig. 6 shows that the assumption of a black-body spectrum allows us to constrain as many point-like sources as wanted, provided that the number of wavelengths is large enough. For instance, $N_{\lambda}=10$ allows us to constrain 9 sources with the visibility amplitudes and 14 if phases are also available.

In conclusion, a multi-wavelength approach allows us to fit more parameters and therefore to distinguish between different scenarios (disc, multiple system, etc.)

\section{Application to circumstellar discs}

Circumstellar discs are a good target for interferometers because they scale from a few tens of AU (where they mostly emit thermal light in the infrared) to a few hundreds of AU (where they present scattered light in the infrared) at a typical distance of $150 \mathrm{pc}$ or more. Two issues of interest are: observing their thermal light, because it comes from the first AUs from the star where planets are supposed to form, and deriving their radial temperature law, because it appears as a good diagnosis of the phenomena involved (irradiation, flaring, viscous dissipation, etc.). In Sect. 3.1 we show how to take into account both the thermal and scattered light, which happen to present different interferometric signatures. In Sect. 3.2, we establish a connection between the temperature law and the wavelength dependence of the visibility.

\subsection{Stellar, thermal and scattered light}

Describing an accretion disc as a marginally resolved object is inaccurate because thermal light occurs at a large scale and accounts for up to $10 \%$ of the total flux. In order to keep the above formalism, we split the image into three components: stellar contribution, thermal emission of the disc, and scattered light. To each contribution, one can associate a corresponding visibility:

$V_{\star}=1-2 \pi^{2} D_{\star}^{2} u^{2}$,

$V_{\mathrm{th}}=\left(1-2 \pi^{2} D_{\mathrm{th}}^{2} u^{2}\right) \exp \left(i \frac{4}{3} S_{\mathrm{th}} D_{\mathrm{th}}^{3} u^{3}\right)$,

$V_{\mathrm{s}}= \begin{cases}1 & \text { if } u=0 \\ 0 & \text { otherwise }\end{cases}$

where $D_{\star}$ is the mean diameter of the star, $D_{\text {th }}$ that of the thermal emission of the disc, and $S_{\text {th }}$ the skewness of distribution of the thermal emission.

We assumed that both the star and the thermal emission of the disc are marginally resolved, that the scattering emission is fully resolved as soon as the baseline is non-zero, and that the star is too small and symmetric to present a phase. Within the approximation that all components have the same photocentre, we derive the total visibility

$V_{\mathrm{tot}}=\frac{F_{\star} V_{\star}+F_{\mathrm{th}} V_{\mathrm{th}}+F_{\mathrm{s}} V_{\mathrm{s}}}{F_{\mathrm{tot}}}$ 
where $F_{\text {tot }}$ is the total flux, $F_{\star}$ that of the star, $F_{\text {th }}$ that of the thermal emission, and $F_{\mathrm{s}}$ that of scattered light. Figure 7 presents a schematic view of the visibility curve for an accretion disc and compares it to that of the thermal light alone. It appears that one point of visibility is not enough to derive the diameter of the disc, as authors usually do, when either the star or scattered flux are present.

\subsection{Temperature profile}

From visibilities at different wavelengths, Malbet \& Berger (2002a) showed that the temperature profile of an accretion disc can be derived. In the context of a massive disc, the flux is dominated by thermal light so that

$1-|V(\lambda)|^{2}=(2 \pi D(\lambda) B / \lambda)^{2}$.

To solve this problem, we need a link between the disc extent $D(\lambda)$ and the temperature profile. The disc extent is given by the second-order moment $\boldsymbol{M}_{2}^{\prime}$ determined from the radial flux distribution of the disc

$F(r)=B_{\lambda}(T(r))$.

We chose a self-similar solution for the sake of simplicity

$T(r) \propto r^{-q}$.

For a disc presenting an inclination $i$, the components of the moments then write (see Appendix $\mathrm{C}$ for a demonstration):

$M_{2}^{\prime 0}=M_{2}^{\prime 2} \cos ^{2} i$

$M_{2}^{\prime 1}=0$,

$M_{2}^{\prime 2} \propto \lambda^{2 / q}$,

so that

$$
\begin{aligned}
D(\lambda) & \propto \lambda^{2 / q}, \\
1-|V|^{2} & \propto \lambda^{2 / q-2} .
\end{aligned}
$$

Using two close enough wavelengths $\lambda_{1}$ and $\lambda_{2}$, we deduce an estimate of $q$ :

$$
q=\frac{1}{1+\frac{1}{2} \frac{\log \left[\left(1-\left|V_{1}\right|^{2}\right) /\left(1-\left|V_{2}\right|^{2}\right)\right]}{\log \left[\lambda_{2} / \lambda_{1}\right]}} .
$$

This result appears as a particular case of the multi-wavelength approach (see Sect. 2.3), that allows us to fit more parameters than one visibility at a single wavelength does. Malbet \& Berger (2002a) obtained a similar result by a more empirical argument: they state that the apparent diameter of a disc is proportional to the radius at which $B_{\lambda}(T(r))$ is maximal. It actually conceals the self-similarity.

Note that the result no longer holds when the disc presents an inner hole. Figure 8 displays the value $q_{\text {app }}$ deduced from Eq. (31) with the $H$ and $K$ bands for a typical FU Ori disc. The parameters of the disc model are given in Appendix D. When the inner gap becomes larger than a few stellar radii, the error on $q,\left|q_{\text {app }}-q\right|$ can be larger than 0.1. Malbet \& Berger (2002a) find $q_{\text {app }} \approx 0.64$ for FU Ori; with a typical value
$R_{*}=6 R_{\odot}$ (Lachaume et al. 2003), we can estimate $q \approx 0.5$ from the curves.

The stellar radius has also an influence because of the unresolved stellar flux, yet, it remains small for FU Ori discs (see Fig. 8). In the case of a T Tauri star, the error could be much larger, because the contribution of the star to the total flux becomes important.

\section{Conclusion}

We have developed a formalism that connects the visibility amplitude and phase of a marginally resolved object with its geometry, namely the moments of the flux distribution. It can prove particularly useful when constraining models that present analytical moments and allows us to retrieve modelindependent spatial information in all cases. It also establishes that the closure phase of a marginally resolved source is a thirdorder term and the visibility a second-order one; therefore, the phase is much harder to detect than the drop in visibility amplitude.

From the formalism, we were also able to estimate the number of parameters relevantly fitted to interferometric measurements. Unless observations are carried out at several wavelengths and the model assumes a black-body-like emission, only a few parameters can be fitted to marginally resolved objects, whatever the number of visibility points taken: three point-like source with visibility amplitudes only, and a fourth one if closure phase is also measured. This limitation is removed when the object is more resolved, that is, if the baselines are longer or if the instrumental accuracy is increased, which allows us to measure the deviation of the visibility and phase from their low-order estimates. This work can therefore be seen as a plea for larger baselines than the CHARA array provides, or high accuracy with IOTA/IONIC or the forthcoming VLTI and Keck.

We then applied this theoretical work to circumstellar discs, by separating the star, the thermal emission of the disc, and the scattered light, the two first ones being well described by their moments. It also allows us to derive, with some hypotheses, information on the radial temperature law in these objects, even if they are underresolved, but requires that measurements should be taken at two or more wavelengths. This can be applied to other field. For instance, limb-darkened stellar photospheres can be probed even with underresolved targets: the equivalent diameter is dependent on the wavelength and one could, with an appropriate model, measure this darkening.

With high precision measurements and/or multiple wavelengths one can access a large number of moments of the flux distribution, which theoretically allows image reconstruction. This is clearly a path that one should investigate in the near future. As a particular case, we believe it is possible to retrieve the radial temperature profile of supposedly symmetrical objects, as as been initiated with FU Ori. The method could also prove useful to constrain the location of stellar spots with high accuracy measurements: the link between the location of these spots and the first order moments of the flux is much clearer than the information given by image reconstruction techniques. 

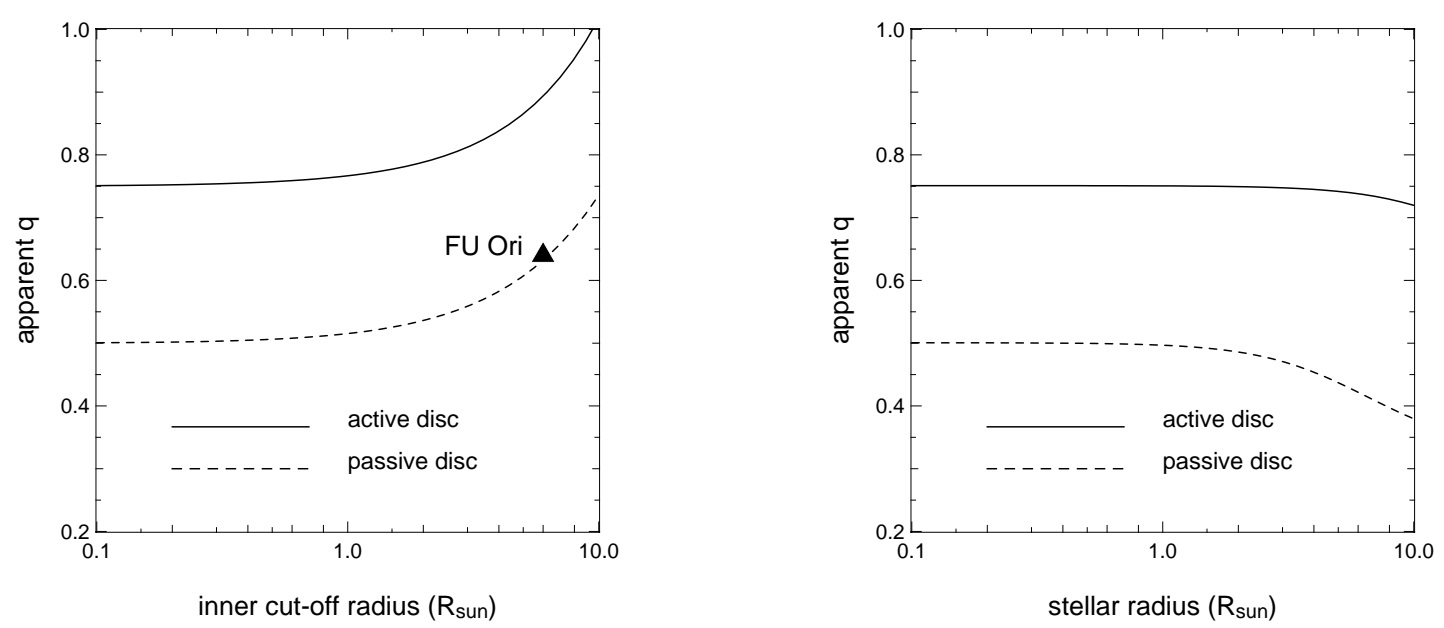

Fig. 8. Apparent temperature law, given by the exponent $q_{\mathrm{app}}$ as a function of the disc inner truncation (left) and the stellar radius (right). Solid line: active disc with $q=0.75$ and an accretion rate of $3 \times 10^{-5} \dot{M} / \mathrm{yr}$; dashed line: flared passive disc with $q=0.5$ and the same effective temperature at $1 \mathrm{AU}$. The star is a black-body at temperature $5000 \mathrm{~K}$. The two visibilities are taken in $H$ and $K$.

Acknowledgements. I thank Fabien Malbet, Jean-Philippe Berger, and Jean-Baptiste Lebouquin for helpful discussions; without their interest, this work would have stayed hidden under a heavy stack on my desk. Computations and graphics have mostly been carried out with the free software Yorick by D. Munro. Useful comments from Theo ten Brummelaar led to an improved presentation of these results.

\section{Appendix A: Moments in Cartesian coordinates}

We use a Cartesian frame in which $\alpha$ has coordinates $(\alpha, \beta)$ throughout this appendix.

The first moment is a vector

$$
\boldsymbol{M}_{1}=\left(M_{1}^{0}, M_{1}^{1}\right)
$$

giving the location of the photocentre in respect to the origin of the frame.

$M_{1}^{0}=\iint I(\alpha, \beta) \alpha \mathrm{d} \alpha \mathrm{d} \beta$,

$M_{1}^{1}=\iint I(\alpha, \beta) \beta \mathrm{d} \alpha \mathrm{d} \beta$.

The second-order moment is a matrix

$\boldsymbol{M}_{2}=\left(\begin{array}{ll}M_{2}^{0} & M_{2}^{1} \\ M_{2}^{1} & M_{2}^{2}\end{array}\right)$

yielding information on the spatial extent of the object. Its components are

$M_{2}^{0}=\iint I(\alpha, \beta) \alpha^{2} \mathrm{~d} \alpha \mathrm{d} \beta$,

$M_{2}^{1}=\iint I(\alpha, \beta) \alpha \beta \mathrm{d} \alpha \mathrm{d} \beta$,

$M_{2}^{2}=\iint I(\alpha, \beta) \beta^{2} \mathrm{~d} \alpha \mathrm{d} \beta$.

The $n$th order moment is a tensor of order $n$ containing $2^{n}$ elements. Since it is symmetrical it is given by only $n+1$ terms, that we note $M_{n}^{k}$ for $0 \leq k \leq n$ :

$M_{n}^{k}=\iint I(\alpha, \beta) \alpha^{n-k} \beta^{k} \mathrm{~d} \alpha \mathrm{d} \beta$

\section{Appendix B: $V$ and $\phi$ series development}

We define

$J_{1}=\boldsymbol{M}_{n} \cdot \boldsymbol{u}$,
$J_{n}=\boldsymbol{M}_{n}^{\prime} \cdot \underbrace{\boldsymbol{u} \cdots \boldsymbol{u}}_{n \text { times }}, \quad$ for $n \geq 2$.

The visibility amplitude and phase are then expressed as:

$$
\begin{aligned}
V= & 1-4 \pi^{2} J_{2}+\frac{4 \pi^{4}}{3}\left(J_{4}+3 J_{2}^{2}\right) \\
& -\frac{8 \pi^{6}}{45}\left(J_{6}-10 J_{3}^{2}+15 J_{4} J_{2}\right) \\
& +\frac{4 \pi^{8}}{315}\left(J_{8}+35 J_{4}^{2}-56 J_{3} J_{5}+28 J_{6} J_{2}\right) \\
& +O\left(u^{10}\right) \\
\varphi= & 2 \pi J_{1}-\frac{4 \pi^{3}}{3} J_{3}+\frac{4 \pi^{5}}{15}\left(J_{5}-10 J_{3} J_{2}\right) \\
& -\frac{8 \pi^{7}}{315}\left(J_{7}+219 J_{3} J_{2}^{2}-21 J_{2} J_{5}-35 J_{3} J_{5}\right) \\
& +\frac{4 \pi^{9}}{2835}\left(J_{9}+2520 J_{3} J_{2} J_{4}+560 J_{3}^{3}-36 J_{7} J_{2}\right. \\
& \left.-84 J_{3} J_{6}-126 J_{5} J_{4}+756 J_{5} J_{2}^{2}-7560 J_{3} J_{2}^{3}\right) \\
& +O\left(u^{11}\right) .
\end{aligned}
$$

\section{Appendix C: Moments of a self-similar disc}

We consider a face-on disc with a radial temperature law $T(r) \propto$ $r^{-q}$, where $r$ is the angular distance from the centre. The flux distribution then reads

$F(r)=\frac{C_{1}}{\lambda^{5}\left(\exp \frac{C_{2}}{\lambda r^{-q}}-1\right)}$,

where $C_{1}$ and $C_{2}$ are constants. The reduced moments are

$M_{2}^{\prime 0}=\frac{1}{F} \iint F(x, y) x^{2} \mathrm{~d} x \mathrm{~d} y$, 
$M_{2}^{\prime 1}=\frac{1}{F} \iint F(x, y) x y \mathrm{~d} x \mathrm{~d} y$

$M_{2}^{\prime 2}=\frac{1}{F} \iint F(x, y) y^{2} \mathrm{~d} x \mathrm{~d} y$,

where $F$ is the total flux given by

$F=\int F(r) 2 \pi r \mathrm{~d} r$.

For the sake of simplicity, we introduce the integral

$I_{s}=\int F(r) 2 \pi r^{s} \mathrm{~d} r$.

By switching to polar coordinates in Eq. (C.2) + Eq. (C.4) and with the help of symmetry, we derive

$M_{2}^{\prime 0}=I_{3} /\left(2 I_{1}\right)$,

$M_{2}^{\prime 1}=0$,

$M_{2}^{\prime 2}=I_{3} /\left(2 I_{1}\right)$.

We perform the change of variables $u=C_{2} /\left(\lambda r^{-q}\right)$ in Eq. (C.6) to find

$I_{s} \propto \lambda^{s / q-4}$.

So,

$M_{2}^{\prime 0}=M_{2}^{\prime 2} \propto \lambda^{2 / q}$.

Since $M_{2}^{\prime}=0$,

$1-|V|^{2}=\frac{M_{2}^{\prime 0} B_{u}^{2}+M_{2}^{\prime 2} B_{v}^{2}}{\lambda^{2}}$,

where $\left(B_{u}, B_{v}\right)=\lambda \boldsymbol{u}$ is the projected baseline. Therefore

$1-|V|^{2} \propto \lambda^{2 / q-2}$.

If the disc is not face-on, the second-order moment along the minor axis is shrinked by a factor $\cos ^{2} i$, where $i$ is the inclination; but the above demonstration still holds.

\section{Appendix D: Power-law accretion disc model}

The FU Ori disc model has been determined with an effective temperature $T(r)=K r^{-q}$. The influence of the central gap or of the star highly depends on the constant $K$. In the standard viscous disc model ( $q=3 / 4)$ by Shakura $\&$ Sunyaev (1973),

$K=\sqrt[4]{\frac{3 G M_{*} \dot{M}}{8 \sigma \pi}}$, where $M_{*}$ stands for the mass of the star and $\dot{M}$ for the accretion rate. If a deviation from $q=3 / 4$ is observed, we should carry out a proper modelling of the involved phenomena. For the sake of simplicity, we assumed that $K$ is always determined by the viscous temperature at $1 \mathrm{AU}$, so that

$T(r)=\frac{K}{(1 \mathrm{AU})^{-q}}\left(\frac{r}{1 \mathrm{AU}}\right)^{-q}$.

We took

$\begin{aligned} M_{*} & =1 M_{\odot}, \\ \dot{M} & =3 \times 10^{-5} M_{\odot} / \mathrm{yr} .\end{aligned}$

\section{References}

Akeson, R. L., Ciardi, D. R., van Belle, G. T., \& Creech-Eakman, M. J. 2002, ApJ, 566, 1124

Akeson, R. L., Ciardi, D. R., van Belle, G. T., Creech-Eakman, M. J., \& Lada, E. A. 2000, ApJ, 543, 313

Armstrong, J. T., Mozurkewich, D., Rickard, L. J., et al. 1998, ApJ, 496, 550

Carleton, N. P., Traub, W. A., Lacasse, M. G., et al. 1994, in Amplitude and Intensity Spatial Interferometry II, ed. J. B. Breckinridge, Proc. SPIE, 2200, 152

Colavita, M. 2001, Am. Astron. Soc. Meeting, 198

Colavita, M. M., Wallace, J. K., Hines, B. E., et al. 1999, ApJ, 510, 505

Glindemann, A., Abuter, R., Carbognani, F., et al. 2000, in Interferometry in Optical Astronomy, ed. P. J. Lena, \& A. Quirrenbach, Proc. SPIE, 4006, 2

Hanbury Brown, R., Davis, J., \& Allen, L. R. 1974, MNRAS, 167, 121

Haniff, C. A., Baldwin, J. E., Boysen, R. C., et al. 2000, in Interferometry in Optical Astronomy, ed. P. J. Lena, \& A. Quirrenbach, Proc. SPIE, 4006, 627

Labeyrie, A. 1975, ApJ, 196, 71

Lachaume, R., Malbet, F., \& Monin, J.-L. 2003, 400, 185

Malbet, F., \& Berger, J.-P. 2002a, Proc. SPIE, in press

Malbet, F., \& Berger, J.-P. 2002b, in SF2A - Scientific Highlights 2001, ed. F. Combes, D. Barret and F. Thévenin (EDP Sciences), 457-460

Malbet, F., Berger, J.-P., Colavita, M. M., et al. 1998, ApJ, 507, 149

Michelson, A. A. 1891, PASP, 3, 274

Michelson, A. A. 1920, ApJ, 51, 257

Michelson, A. A., \& Pease, F. G. 1921, ApJ, 53, 249

Monnier, J. D., \& Millan-Gabet, R. 2002, ApJ, 579, 694

Shakura, N. I., \& Sunyaev, R. A. 1973, A\&A, 24, 337

ten Brummelaar, T. A., Bagnuolo, W. G., McAlister, H. A., et al. 2000, in Interferometry in Optical Astronomy, ed. P. J. Lena, \& A. Quirrenbach, Proc. SPIE, 4006, 564 